\title{
MARCKS Protein Inhibitor BIO-11006
}

National Cancer Institute

\section{Source}

National Cancer Institute. MARCKS Protein Inhibitor BIO-11006. NCI Thesaurus. Code C156733.

An aerosolized 10-amino acid peptide that inhibits the myristoylated alanine rich protein kinase C substrate (MARCKS) protein, with potential immunomodulating and antineoplastic activities. Upon inhalation, the MARCKS protein inhibitor BIO-11006 targets, binds to and inhibits the phosphorylation of MARCKS (P-MARCKS). This prevents MARCKS-mediated signaling, thereby preventing the release of phosphatidylinositol 4,5bisphosphate (PIP2) from the cell membrane upon MARCKS binding. This prevents the PIP2-mediated activation of focal adhesion kinase (FAK) and the FAK-mediated activation of the PI3K/AKT pathway and the activation of integ rins, talin, vinculin and paxillin. This leads to an inhibition of tumor cell proliferation, migration, metastasis and survival. In addition, inhibition of MARCKS prevents mucin granule release and reduces the overproduction of mucus in the lungs. This may abrogate airway obstruction, impaired lung function, airway inflammation and bacterial infections associated with overproduction of mucus in the lungs. The MARCKS protein, a filamentous actin crosslinking protein and substrate for protein kinase $C(P K C)$ is localized on the plasma membrane. Upon phosphorylation by PKC or binding to the calcium-calmodulin complex, the association of MARCKS with actin and with the plasma membrane is blocked, leading to its presence in the cytoplasm. The MARCKS protein plays a key role in the exocytosis of a number of vesicles and granules, cell movement, mitogenesis and membrane trafficking. 